\title{
Yeast "Knockout-and-Rescue" System for Identification of eIF4E-Family Members Possessing eIF4E-Activity
}

BioTechniques 33:392-401 (August 2002)

\author{
Bhavesh Joshi, Javier \\ Robalino, Eric J. Schott, and \\ Rosemary Jagus \\ University of Maryland \\ Biotechnology Institute, \\ Baltimore, MD, USA
}

\begin{abstract}
Evidence from several laboratories and sequencing projects has revealed that many eukaryotes contain multiple proteins related in sequence to the human mRNA-cap binding translation initiation factor $4 E$ (eIF4E1). Although some have been shown to bind cap-analogues, whether all eIF4E-family members function as translation initiation factors is unclear. Furthermore, the existence of proteins related to eIF4E complicates the identification of the translation factor by sequence-based approaches. Methods to assess the functionality of eIF4E are limited. The most informative, single assay to identify proteins with eIF4E-activity is that of rescue of the lethal disruption of the single Saccharomyces cerevisiae eIF4E gene. We have developed a simplified yeast eIF4E "knockout-and-rescue" system, the characteristics of which are (i) a haploid system that obviates the need for a "plasmid shuffle", (ii) a simple G418-based selection for yeast lacking a chromosomal eIF4E gene, and (iii) a glucose-based selection to deplete the strain of a human eIF4E-1 substitute and to assess the eIF4E-activity of an untested eIF4E-family member. In this form, the yeast eIF4E knockout-and-rescue system becomes a tool available to any laboratory experienced in the selection of microbial strains with antibiotics and standard media for the identification and isolation of $c D N A s$ encoding proteins with eIF4E-activity.
\end{abstract}

\section{INTRODUCTION}

The initiation of translation of most eukaryotic mRNAs involves their recruitment to the ribosomal apparatus through the activity of the translation initiation factor eIF4E, which binds to the $5^{\prime} \mathrm{m}^{7} \mathrm{GTP}$-cap structure of mRNAs and the initiation factor eIF4G $(10,17)$. The Saccharomyces cerevisiae eIF4E gene was shown, by gene disruption, to be the only eIF4E counterpart in this yeast (1), a fact that has since been confirmed from knowledge of the complete $S$. cerevisiae genome (http://www. mips.biochem.mpg.de). With the exception of plant cells, which have long been known to contain at least two different proteins with activities similar to mammalian eIF4E-1 (5), this evidence suggested that most organisms contain a single eIF4E gene. However, recent evidence from a number of laboratories, including our own, and from genome sequencing projects has revealed that organisms of both plant and animal kingdoms contain a number of related proteins $(11-13,16,18,21,22)$. Together eIF4E and its relatives com prise a family of eIF4E-like proteins within a particular organism.

With the exception of eIF4E, the functions of each family member are poorly understood. Although some have been shown to bind cap-analogs in vitro $(11,12,16,18,21,22)$, whether all members function as translation initiation factors, whether some act as competitive inhibitors of eIF4E, or even serve functions unrelated to those of eIF4E is unclear. Furthermore, the existence of proteins related to eIF4E complicates the identification of the translation factor by sequence-based approaches. Methods available to as- sess whether an eIF4E-family member can function as an initiation factor are limited. The most informative, single assay to directly identify cDNAs encoding proteins with eIF4E-activity is that of the rescue of the lethal disruption of the single $S$. cerevisiae eIF4E gene by an exogenously expressed eIF4E $(1,20$, $25,27)$. Rescue of growth and translation by expression of an exogenous cDNA in the yeast eIF4E "knockoutand-rescue" system provides evidence that the protein encoded is capable of interacting with components of the yeast translational machinery including yeast eIF4G and mRNAs. Since mam malian eIF4E-1s, which shares only approximately $30 \%$ similarity to the yeast protein, can replace the yeast eIF4E (1), this assay system should be suitable for testing eIF4E-family members from a wide range of organisms.

Previous eIF4E knockout-and-rescue systems have employed auxotrophic markers for the eIF4E gene replacement in either diploid or haploid yeast strains that previously lack this gene $(1,3,20,27)$. The use of diploid strains to assess the function of an untested eIF4E is technically difficult and time consuming, requiring specialized microscopic equipment for, and expertise in, the isolation, separation, and analysis of haploid spores (tetrad analysis). However, the use of haploid strains requires only the use of media selection techniques that are accessible to most laboratories that routinely culture and maintain bacterial stocks.

Haploid strains lacking the genomic yeast eIF4E gene can be generated most simply by disruption of the yeast eIF4E gene in a strain that has been previously transfected with an extrachromosomal plasmid that allows the 
expression of a known, functional eIF4E "substitute", such as the yeast eIF4E or mammalian eIF4E-1. Assessment of the eIF4E-activity of the eIF4E-family member in question can be performed using a technique known as a "plasmid shuffle" (23). In essence, transfection of the "knockout" haploid strain with a plasmid carrying the exogenous cDNA in question is followed by "removal" of the extrachromosomal substitute plasmid by selections in minimal synthetic media, which select against its presence.

An alternative method of using haploids to assess the eIF4E-activity of an eIF4E-family member is through the use of yeast strains lacking a chromosomal eIF4E but carrying a repressible exogenous eIF4E substitute. Two available strategies could be used for this purpose: ( $i$ ) using a yeast strain that carries a temperature-sensitive eIF4E isoform in which growth at the nonpermissive temperature can be rescued by expression of a second eIF4E-family member (1) and (ii) using a yeast strain in which expression of the eIF4E substitute is regulated by the use of the glucose-repressible Gal-promoters and in which growth in the presence of glucose can be accomplished by expression of a cDNA encoding a functional eIF4E under the control of a promoter active in the presence of glucose (2).

In all cases, however, maintenance of the strain lacking an intact chromosomal yeast eIF4E gene and removal of contaminating organisms are accom plished through selection and growth on synthetic media that lack certain amino acids and/or nucleotides. Such media can be both costly and time consuming to prepare.

We have developed an improved haploid yeast eIF4E knockout-and-rescue system that allows a simple G418based selection for yeast lacking a chromosomal eIF4E gene using standard Bacto-yeast/Bacto-peptone (YP)-based media combined with the ease of the glucose-based selection for assessing the ability of cDNAs encoding eIF4Efamily members to rescue growth. In this simplified form, the yeast eIF4E knockout-and-rescue system becomes a tool easily used by any laboratory already experienced in the simple selection of microbial strains with antibiotics and standard media. Here we have applied the system to determine which of two zebrafish proteins that share almost equal similarity to mammalian eIF4E-1 represents a functional translation factor in zebrafish. Our results indicate that only one zebrafish form, eIF4E-1A, is able to rescue growth of the yeast strain lacking a functional eIF4E gene. The other zebrafish protein, eIF4E-1B, appears to be a novel, eIF4E-related protein that lacks the ability to replace eIF4E in yeast. Subsequent analyses have revealed that eIF4E-1B does not interact with the mRNA-cap structure and eIF4G in vitro (18). We have also used the strategy for mutational analyses of eIF4Es $(14,18)$. The yeast eIF4E knockout-and-rescue system can also be adapted for the identification and isolation of cDNAs encoding eIF4E isoforms from cDNA libraries derived from mRNAs of any species.

\section{MATERIALS AND METHODS}

\section{Growth and Maintenance of S. cerevisiae Strains}

The haploid $S$. cerevisiae strain BY 4739 MAT $\alpha$ leu2D0 lys2D0 ura3D0 (4) and its derivatives (Table 1) were grown at $30^{\circ} \mathrm{C}$ in YP media ( $1 \%$ yeast extract, $2 \%$ bactopeptone) containing either $2 \%$ D-glucose or $2 \%$ D-galactose. When needed, G418 was included at $200 \mu \mathrm{g} / \mathrm{mL}$ for the selection. Yeast strains were also grown in standard synthetic medium (SC) containing either $2 \%$ D-glucose (SCDex) or $2 \%$ Dgalactose (SCGal). Selective media were prepared using SC medium lacking nutrients (Ura and/or Leu), as indicated. Transfections of DNA into $S$. cerevisiae were performed as described by Geitz et al. (9).

\section{Polymerase Amplification Conditions}

PCR amplifications for the generation of the gene disruption cassette and detection of genomic DNA fragments were performed under standard "hotstart" conditions using Taq DNA polymerase (Fisher Scientific, Pittsburgh, PA, USA) and primer sets as indicated. Annealing and extension steps were conducted at $52^{\circ} \mathrm{C}$ for $30 \mathrm{~s}$ and $72^{\circ} \mathrm{C}$ for $2 \mathrm{~min}$ for $20-40$ cycles as indicated. Amplified DNA was separated by TAE-agarose gel electrophoresis and stained with ethidium bromide before visualization.

\section{Generation of DNA Constructs Containing cDNAs Encoding eIF4E cDNAs}

The complete human eIF4E-1 cDNA was excised from the plasmid pET11dheIF4E (24) by digestion with the restriction enzymes $\mathrm{Xba \textrm {I }}$ and $\mathrm{BamHI}$ and ligated to the yeast-E. coli shuttle vector pRS415GAL1 (ATCC, Manassas, VA, USA) to generate pRS415GAL1h4E. An identical procedure was employed for the construction of pRS416GPD (15) (ATCC) containing cDNAs encoding zebrafish eIF4E-family members: eIF4E-1A [accession no. AF257519 (19)] and eIF4E-1B $(7,8)$.

\section{Yeast eIF4E Gene (CDC33) Replacement}

The haploid strain BY $4739 \alpha$ was initially transfected with either the vector pRS415GAL1 or pRS415GAL1hum4E, and colonies were selected for growth on SCDex-Ura, generating strains JOS001 and JOS002, respectively. Strain JOS002 was transformed with a 1603-bp G418-resistance cassette generated by PCR ( 20 cycles) from the plasmid pFA6a-KanMX4 (26) using primers FKO (residues -14 to +34 of yeast eIF4E attached to 5'-CGTACGCTGCAGGTCGAC-3') and RKO (+616 to +664 of yeast eIF4E attached to $5^{\prime}$-ATCGATGAATTCGAGCTCG-3') (numbers relative to yeast eIF4E translational start site). To ensure the production of a blunt-ended DNA fragment, VentR DNA polymerase (New England Biolabs, Beverly, MA, USA) was included in the PCR. Following transfection, yeast were selected on YP medium containing G418 and galactose. Resulting colonies were assessed for their ability of grow on YP medium containing G418 and glucose leading to the isolation of strains JOS003 and JOS004 (derived from homologous and nonhomologous recombination, respectively). Disruption of the S. cerevisiae eIF4E gene (CDC33) was verified by PCR analyses using genom ic DNA as a substrate. 


\section{Research Report}

Table 1. Genotypes and Growth Characteristics of Yeast Strain BY 4739 (4) and Derivatives Generated for This Study

\begin{tabular}{|c|c|c|c|c|c|c|}
\hline \multirow[b]{3}{*}{ Strain } & \multirow[b]{3}{*}{ Genotype } & \multirow[b]{3}{*}{ Plasmids } & \multicolumn{4}{|c|}{ Growth in YP Media } \\
\hline & & & \multicolumn{2}{|c|}{ Galactose } & \multicolumn{2}{|c|}{ Glucose } \\
\hline & & & $-G 418$ & +G418 & $-G 418$ & +G418 \\
\hline BY 4739 & MAT $\alpha$ leu2D0 lys2D0 ura3D0 & None & $\sqrt{ }$ & $x$ & $\sqrt{ }$ & $x$ \\
\hline JOS001 & BY 4739 (LEU2) & pRS415GAL1 & $\sqrt{ }$ & $\mathrm{x}$ & $\sqrt{ }$ & $x$ \\
\hline JOS002 & BY 4739 (LEU2 GAL1-helF4E) & pRS415GAL1h4E & $\sqrt{ }$ & $x$ & $\sqrt{ }$ & $x$ \\
\hline JOS003 & JOS002 CDC33::KanMX4 & pRS415GAL1h4E & $\sqrt{ }$ & $\sqrt{ }$ & $x$ & $x$ \\
\hline JOS004 & JOS002 KanMX4 & pRS415GAL1h4E & $\sqrt{ }$ & $\sqrt{ }$ & $\sqrt{ }$ & $\sqrt{ }$ \\
\hline JOS105 & JOS003 (URA3) & pRS415GAL1h4E + pRS416GPD & $\sqrt{ }$ & $\sqrt{ }$ & $x$ & $x$ \\
\hline JOS106 & JOS003 (URA3 GPD-helF4E) & pRS415GAL1h4E + pRS416GPDh4E & $\sqrt{ }$ & $\sqrt{ }$ & $\sqrt{ }$ & $\sqrt{ }$ \\
\hline JOS107 & JOS003 (URA3 GPD-zelF4E-1A) & pRS415GAL1h4E + pRS416GPDz4E-1A & $\sqrt{ }$ & $\sqrt{ }$ & $\sqrt{ }$ & $\sqrt{ }$ \\
\hline JOS108 & JOS003 (URA3 GPD-zelF4E-1B) & pRS415GAL1h4E + pRS416GPDz4E-1B & $\sqrt{ }$ & $\sqrt{ }$ & $x$ & $x$ \\
\hline JOS109 & $\begin{array}{l}\text { BY } 4739 \text { CDC33::KanMX4 } \\
\text { (URA3 GPD-helF4E) }\end{array}$ & pRS416GPDh4E & $\sqrt{ }$ & $\sqrt{ }$ & $\sqrt{ }$ & $\sqrt{ }$ \\
\hline JOS110 & $\begin{array}{l}\text { BY } 4739 \text { CDC33::KanMX4 } \\
\text { (URA3 GPD-zelF4E-1A) }\end{array}$ & pRS416GPDz4E-1A & $\sqrt{ }$ & $\sqrt{ }$ & $\sqrt{ }$ & $\sqrt{ }$ \\
\hline
\end{tabular}




\section{Generation of Yeast Strains Carrying Human and Zebrafish eIF4E Variants}

Transformation of strain JOS003 with pRS416GPD and derivatives of these vectors containing eIF4E variants, followed by selection on SCGALUra-Leu+G418, gave rise to derivative strains JOS105 to JOS108. Strains JOS109 to JOS110 were generated through the removal of the plasmid pRS415GAL1h4E, induced by overnight growth of the cells in SCGALUra+G418, followed by assessment of the inability to grow in SCGAL-UraLeu+G418.

\section{Isolation of Total $S$. cerevisiae Proteins and SDS-PAGE and Western Analysis}

Single $S$. cerevisiae colonies from agar plates were used to inoculate suspension media $(5 \mathrm{~mL})$, as indicated. Following incubation at $30^{\circ} \mathrm{C}$ for $24 \mathrm{~h}$ with shaking, cells from $1 \mathrm{~mL}$ were collected by centrifugation at $2000 \times g$, washed, and resuspended in $0.75 \mathrm{~mL}$ sterile water. Cells were lysed with 1 vol $2 \times$ SDS-PAGE sample buffer before separation of total cellular proteins with $10 \mu \mathrm{L}$ of the suspension by $13 \%$ SDSPAGE. Proteins were electrotransferred to a PVDF membrane in $20 \%$ methanol, $125 \mathrm{mM}$ Tris-glycine, $\mathrm{pH} 8.3,0.01 \%$ SDS, using $60 \mathrm{~V}$ for $1.25 \mathrm{~h}$. The mem brane was blocked with BLOTTO (25 $\mathrm{mM}$ Tris- $\mathrm{HCl}, \mathrm{pH}$ 8.0, $200 \mathrm{mM} \mathrm{NaCl}$, $5 \%$ nonfat milk powder) before incubation with rabbit anti-human eIF4E (a gift from the laboratory of Dr. R.E. Rhoads), HRP-linked goat anti-rabbit IgG (Bio-Rad Laboratories, Hercules, CA, USA) and subsequent detection by enhanced chemiluminesence using the $\mathrm{ECL}^{\circledR}$ reagent system (Amersham Biosciences, Piscataway, NJ, USA).

\section{RESULTS}

\section{Generation of a Simple Yeast Knockout-and-Rescue System}

Our goal was to create a simplified yeast eIF4E knockout-and-rescue system to assess the ability of exogenous eIF4E-family members to function with eIF4E-activity, amenable to use by almost any laboratory familiar with the simple growth and selection of microorganisms with antibiotics. The characteristics of the generated system are $(i)$ a haploid system that obviates the need for a complete plasmid shuffle, (ii) a simple G418-based selection for yeast strains lacking an endogenous chromosomal eIF4E requiring the use of standard YP media, and (iii) a glucose-based media selection to both deplete the yeast strain of a functional eIF4E substitute and allow the assessment of the eIF4E-activity of an untested eIF4E-family member.

Figure 1 outlines the strategy we em ployed to generate a yeast strain lacking an endogenous chromosomal eIF4E gene. Table 1 presents a summary of the genotypes and selected phenotypes of the strains generated during this study. The initial strain chosen was that of the haploid S. cerevisiae strain BY $4739 \alpha$ (leu2D0 lys2D0 ura3D0) (4). This strain was generated by targeted gene disruption of the LEU2, LYS2, and URA3 genes, thus minimizing reversions that occur in other strains. Initially, strain BY $4739 \alpha$ was transformed with the stable single-copy plasmid pRS415GAL1h4E containing cDNA encoding human eIF4E, downstream of the glucose-repressible, galactose-dependent GAL1 promoter and the LEU2-selectable marker. Following selection on medium lacking Leu, yeast colonies were grown and assessed for their ability to express human eIF4E-1 in the presence of either glucose or galactose (Figure 2). Strains containing the control vector pRS415GAL1 (without eIF4E-1 cDNA) were also assessed (Figure 2, lanes 2 and 3). In the presence of glucose, human eIF4E-1 expression from the pRS415GAL1h4E construct was repressed, and, thus, little or no human eIF4E-1 was detected (Figure 2, lane 4). However, in the presence of galactose and absence of glucose, human eIF4E-1 expression was induced (Figure 2, lane 5). The presence of exogenous human eIF4E-1 did not affect survival of wild-type yeast compared to yeast transfected with control pRS415GAL1 plasmid, suggesting that expression of human eIF4E-1 was not inhibitory to yeast growth (data not shown).

The yeast strain expressing human eIF4E-1 was further transformed with a 
linear PCR-generated DNA fragment consisting of the G418-resistance cassette, KanMX4, surrounded by 40 bp each of $5^{\prime}$ and $3^{\prime}$ sequences of the $S$. cerevisiae eIF4E gene. Transformed yeast were selected on YP medium containing G418 and galactose. A depiction of the result of the expected homologous recombination is presented in Figure 3A. G418-resistant strains were further selected for their ability to grow in glucose or galactose. Assessment of the ability of selected strains to grow on media containing G418 and either galactose or glucose are presented in Table 1 and Figure 5. As can be seen, unlike control strains JOS001 and JOS002, strain JOS003 is resistant to G418 and is able to grow in the presence of galactose but not in the presence of glucose, suggesting that it is the product of homologous recombination and gene replacement. Strain JOS004 (Table 1) is able to grow in media containing either glucose or galactose, suggesting it is the product of nonhomologous recombination and still carries an intact chromosomal $S$. cerevisiae eIF4E gene.

Disruption and gene replacement were confirmed by PCR analyses (Figure 3, B and C). PCR analyses reveal the presence of an intact yeast eIF4E gene in the DNA of control strain JOSO02, but not in the DNA of strain JOSO03 (Figure $3 B$ ). Primer sets to assess the presence of the G418-resistance cassette revealed that strain JOS003 indeed contained a disrupted eIF4E gene in the manner expected following a homologous recom bination event (Figure 3C). PCR analysis of DNA from strain JOS004 confirmed that this strain carries an intact chromosomal eIF4E gene and gained G418-resistance through nonhomologous recombination (data not shown). Since strain JOS003 lacks the

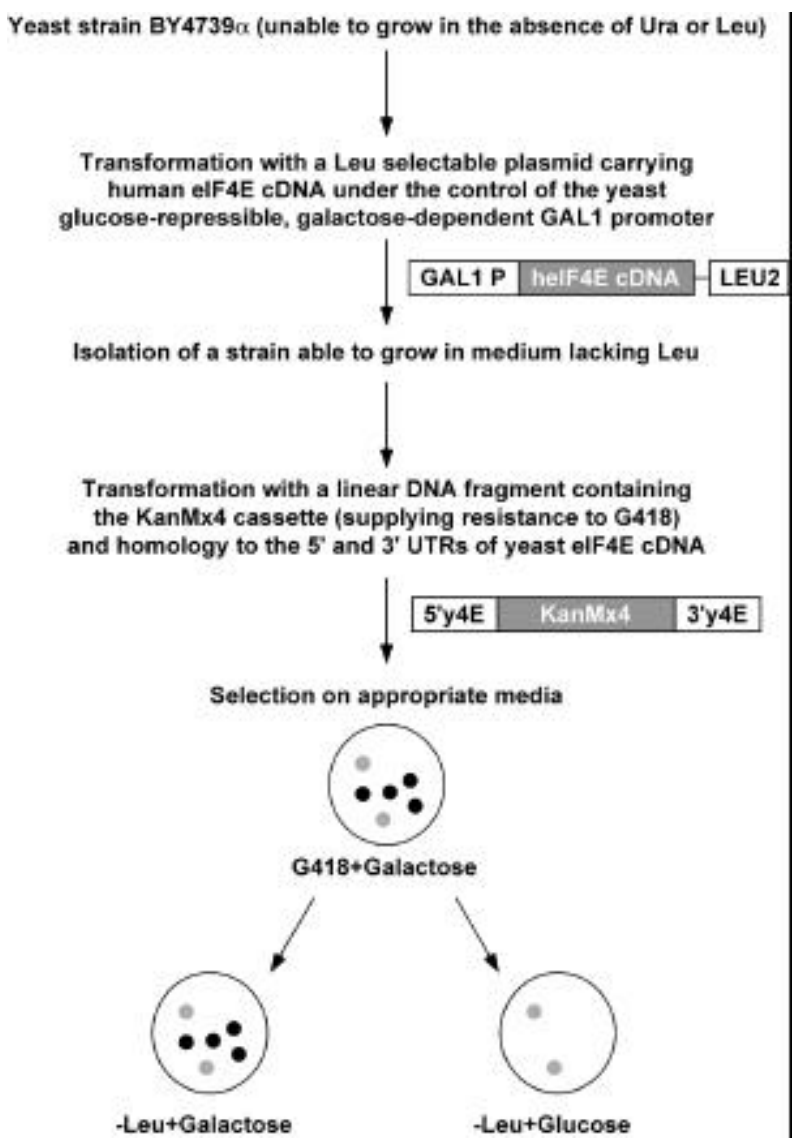

Figure 1. Scheme for the generation of a haploid G418-resistant, galactose-dependent, glucose-sensitive yeast strain lacking a chromosomal eIF4E gene. Black and gray spots, derivatives of homologous and nonhomologous recombinations, respectively. ability to grow in glucose because of the repression of the GAL1 promoter, the strain can be used directly to assess the activity of exogenous eIF4Es from other species by their expression under the control of a promoter active in glucose.

\section{Identification of eIF4E-Family Members with eIF4E-Activity Using the Yeast System}

To illustrate how this system may be used, we assessed the capacity of two zebrafish cDNAs encoding eIF4E-family members of almost equal identity and similarity to the functional human eIF4E-1, to rescue growth of the strain in media containing glucose. Initial cloning of a zebrafish cDNA encoding a protein related to mammalian eIF4E-1, using degenerate primers and RT-PCR, suggested that zebrafish express a protein, originally termed zeIF4EL (7), of similar size and $66 \%$ identity to human eIF4E. How ever, sequences in the EST cDNA bank [AA542678 (6)] suggest the presence of a different mRNA encoding a zebrafish eIF4E-1 form, with greater homology ( $82 \%$ identity) to human eIF4E-1. We developed a complete cDNA encoding

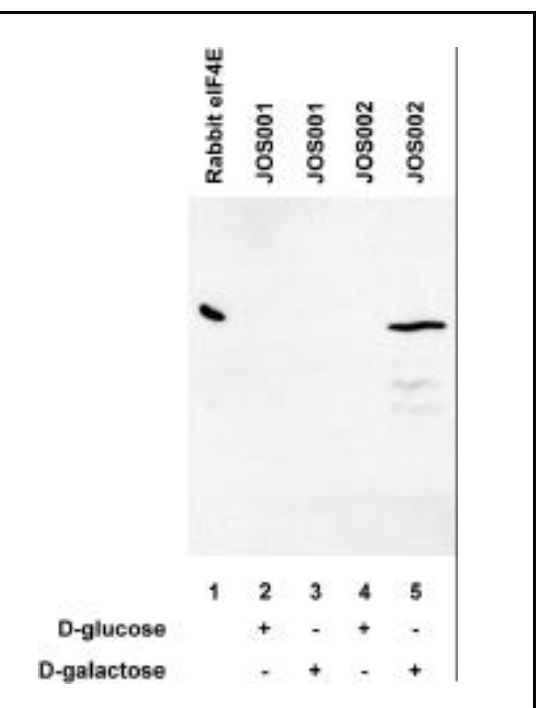

Figure 2. Analysis of the glucose-repression and galactose-dependence of exogenous human eIF4E-1 expression in yeast strains. Yeast BY$4739 \alpha$ strains JOS001 and JOSO02 were cultured overnight from colonies in YP media containing either D-glucose or D-galactose $(5 \mathrm{~mL})$, as indicated. Yeast proteins (equivalent to $0.75 \%$ of cells of the culture) were separated by SDS-PAGE and electrotransferred to a PVDF membrane before visualization of mammalian eIF4E by immunoblotting (lanes 2-5). Lane 1, 1 ng rabbit eIF4E-1. 


\section{Research Report}

this form [accession no. AF257519 (19)]. Based on their relative identity to human eIF4E-1, the two zebrafish eIF4E-family members are termed eIF4E-1B and eIF4E-1A, respectively (Figure 4). Both zebrafish forms share all known amino acids thought to be required for interaction with the mRNAcap structure and binding to eIF4G (10).

cDNAs encoding human eIF4E and zebrafish eIF4E-1A and eIF4E-1B were independently transferred into the Uraselectable plasmid pRS416GPD for expression in yeast under the control of the glucose-active GPD promoter for transfection into the yeast strain JOS003. Following selection of yeast able to grow in SCGal-Ura-Leu, the resulting strains were plated on media containing glucose to assess their ability to rescue growth under a condition in which eIF4E was lacking (Figure 5). As expected, transformation of JOS003 with a vector encoding human eIF4E-1 resulted in yeast that were able to grow in media containing either galactose or glucose (JOS105 and JOS109). Similar results were obtained following transfection and selection of strains containing the vector encoding zebrafish eIF4E1A (JOS107 and JOS110). Interestingly, the strain containing vector encoding eIF4E-1B (JOS108) was unable to rescue growth in glucose. These data suggest that, although both zebrafish eIF4Efamily members share all known amino acids required for activity, only zebrafish eIF4E-1A is functionally similar to mammalian eIF4E-1. That expression of eIF4E-1B fails to rescue the growth of yeast lacking in eIF4E indicates that eIF4E-1B is deficient in the ability to interact with either the mRNA cap-structure or eIF4G. Subsequent analyses have confirmed that eIF4E-1A, but not eIF4E-1B, can interact with the mRNA cap-structure and bind to eIF4G in vitro (18). Consequently, eIF4E-1B represents a novel eIF4E-family member whose function has yet to be discerned.

\section{DISCUSSION}

We have generated a $S$. cerevisiae strain that lacks endogenous chromosomal eIF4E, which is dependent on the glucose-repressed and galactose-dependent expression of human eIF4E-1 for
A

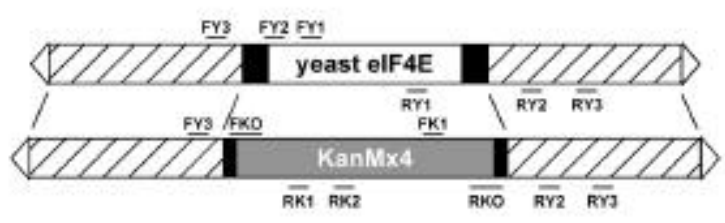

B

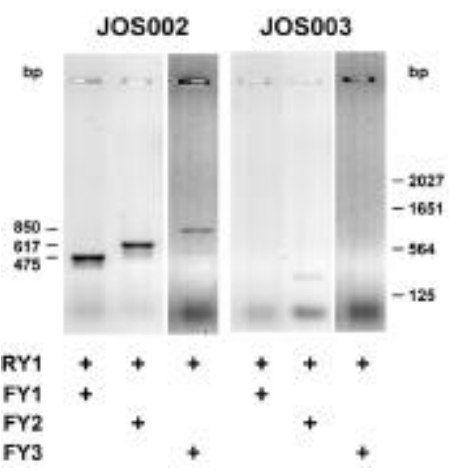

C
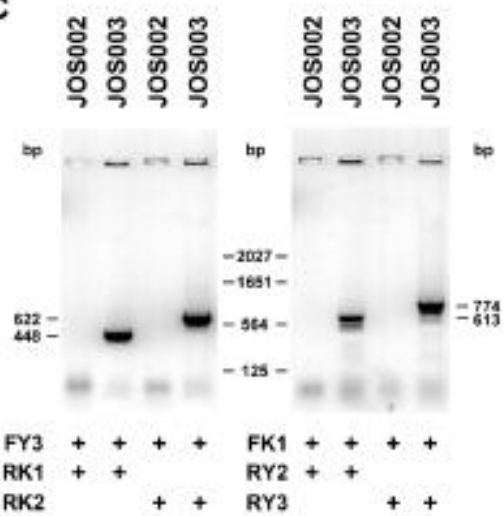

Figure 3. Generation of a yeast strain containing a disrupted genomic eIF4E gene. (A) A schematic showing the wild-type yeast eIF4E gene (cdc33, approximately $0.7 \mathrm{~kb}$ ) and the result of gene disruption following insertion by homologous recombination of a 1.5-kb KanMx4-resistance cassette (not to scale). Primers: FY1(+148 to +168), FY2(+6 to +24$)$, FY3(-228 to -209$)$, RY1(+622 to +600$)$, RY2(+1076 to $+1056)$, RY3(+1237 to +1218), FK1(+1389 to +1408), RK1(+220 to 201), and RK2(+394 to +375). (Note that all numbers are relative to the yeast eIF4E translation start site). (B and C) PCR analysis of genomic DNA derived from strains JOS002 and JOS003 (primers as indicated).

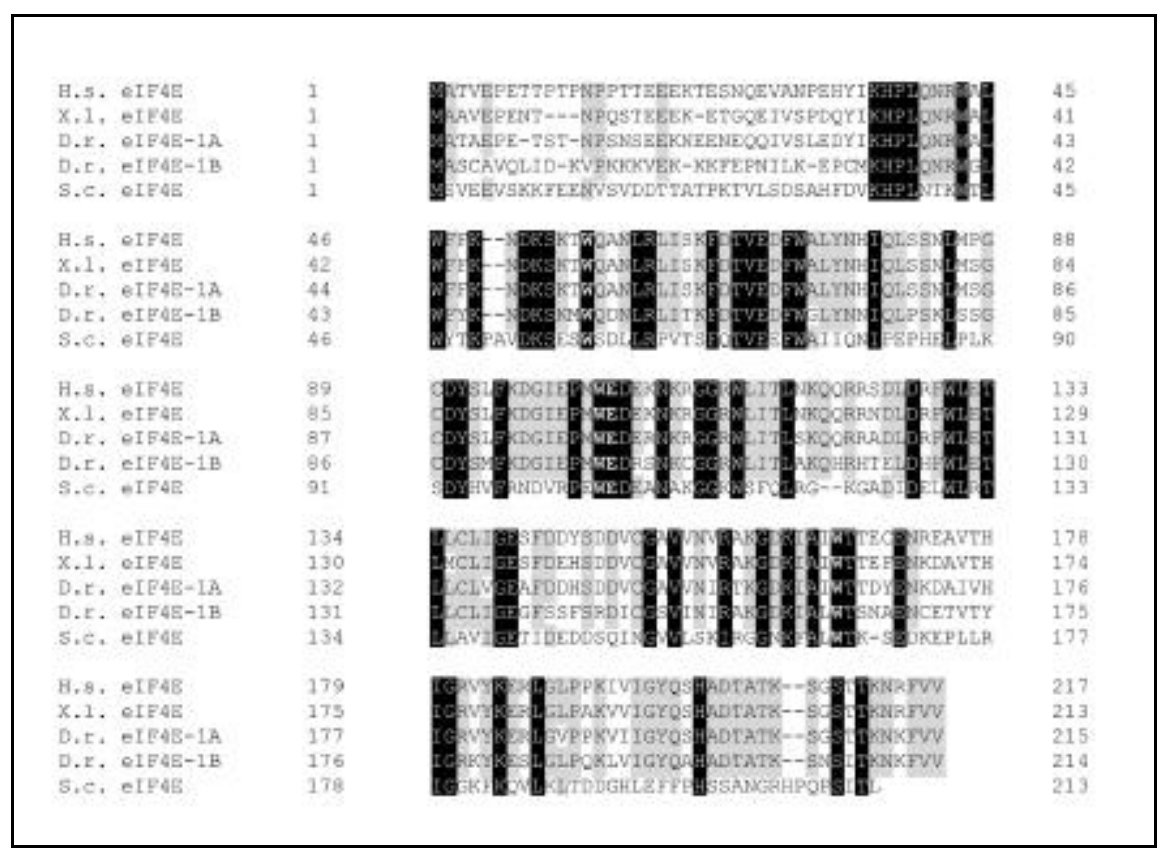

Figure 4. Amino acid sequence alignment of zebrafish eIF4Es with known archetypal eIF4Es of human, Xenopus, and yeast. Black boxes, amino acids identical in all sequences listed. Gray boxes, amino acids identical in $80 \%$ of sequences listed. Bold typeface, residues demonstrated to be important for cap-binding. 


\section{Research Report}

its survival and can be simply selected for by growth in standard YP media containing G418 and galactose and lacking glucose. This strain provides a simple tool to isolate cDNAs encoding eIF4Efamily members with eIF4E-activity from any organism. Since the production of human eIF4E-1 in this system is galactose-dependent and undetectable in medium containing glucose, this strain allows the assessment of functionality of any "test" cDNA encoding an eIF4Efamily member from any organism, following its expression from a plasmid under the control of a promoter that is active in glucose and simple selection on medium containing glucose, obviating the necessity of removing the human eIF4E-1 substitute cDNA by the use of a plasmid shuffle. With the number of $\mathrm{cD}$ NAs encoding proteins related to eIF4E within the same species increasing, it is becoming increasingly difficult to define eIF4E on the basis of sequence similari- ty alone. The simplified yeast eIF4E knockout-and-rescue system described here allows a functional assessment of any cDNA described. Furthermore, this system provides a simple procedure available to any laboratory already familiar with growth and selection of microorganisms to isolate and screen for

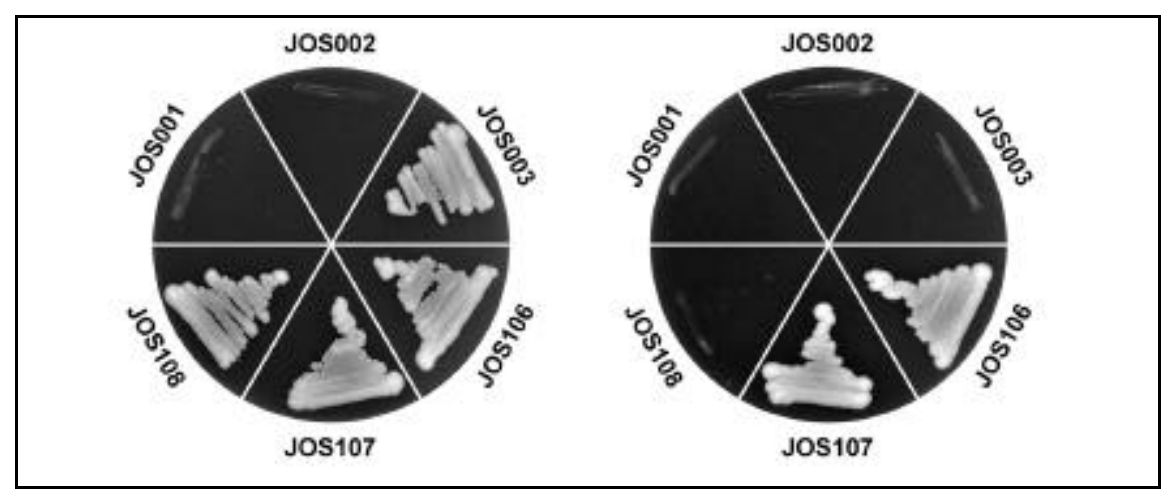

Figure 5. Assessment of the ability of human and zebrafish eIF4Es to rescue the growth $S$. cerevisiae JOS003 in media containing glucose. The $S$. cerevisiae strain, JOS003, was transformed with no vector, the Ura-selectable vector pRS416GPD containing cDNA encoding human eIF4E-1 (JOS106), zebrafish eIF4E-1A (JOS107), or zebrafish eIF4E-1B (JOS108), as indicated. Following selection on SC Gal-U-L media, yeast from the resulting single colonies and control strains JOS001 and JOS002 were transferred to YP-agar media containing G418 and either D-galactose (left plate) or D-glucose (right plate). Growth was assessed following incubation at $30^{\circ} \mathrm{C}$ for $72 \mathrm{~h}$. 
cDNAs encoding eIF4E-family mem bers that can function in yeast from cDNA libraries of any organism.

We have used the system to identify zebrafish eIF4E-1A as the functional equivalent of human eIF4E-1, as well as to demonstrate, through the nonfunctionality of the eIF4E-related protein, eIF4E-1B, that assumptions on function cannot be simply drawn from amino acid sequence alone. We have also used the system to assess the activities of eIF4E variants containing amino acid substitutions (14), deletions, and additions (18).

The yeast knockout-and-rescue system should be suitable for testing eIF4E-family members from a wide range of organisms, since both mam malian and plant eIF4Es, which share only approximately $30 \%$ similarity to the yeast protein, can replace the yeast eIF4E $(1,20)$. However, no single assay can be expected to constitute a standalone test to identify eIF4E molecules. False positives could arise from eIF4Elike proteins that do not function as initiation factors in their species of origin but do interact sufficiently with the yeast translational machinery. Similarly, the absence of complementation does not by itself rule out the possibility that an eIF4E-like protein functions as an initiation factor. False negatives could arise because a cDNA fails to be expressed or because the protein cannot function in the heterologous system. However, the simplicity and versatility of the yeast system provides an informative initial approach to the identification of cDNAs encoding functional eIF4Es before more definitive assessment of function from assays for eIF4E activity such as the ability to bind cap-analogs and eIF4G(s) from relevant species.

\section{ACKNOWLEDGMENTS}

This work was supported by National Science Foundation grant no. MCB9808401 to R.J. and B.J.

\section{REFERENCES}

1.Altmann, M., P.P. Muller, J. Pelletier, N. Sonenberg, and H. Trachsel. 1989. A mam malian translation initiation factor can substitute for its yeast homologue in vivo. J. Biol.
Chem. 264:12145-12147.

2.Altmann, M., N. Sonenberg, and H. Trachsel. 1989. Translation in Saccharomyces cerevisiae: initiation factor 4E-dependent cell-free system. Mol. Cell. Biol. 9:4467-4472.

3.Altmann, M. and H. Trachsel. 1997. Translation initiation factor-dependent extracts from yeast Saccharomyces cerevisiae. Methods 11:343-352.

4.Brachmann, C.B., A. Davies, G.J. Cost, E. Caputo, J. Li, P. Hieter, and J.D. Boeke. 1998. Designer deletion strains derived from Saccharomyces cerevisiae S288C: a useful set of strains and plasmids for PCR-mediated gene disruption and other applications. Yeast 14:115-132

5.Browning, K.S., S.R. Lax, and J.M. Ravel. 1987. Identification of two messenger RNA cap binding proteins in wheat germ. J. Biol. Chem. 262:11228-11232.

6.Clark, M., H. Lehrach, B. Appel, J. Eisen, S. Johnson, M. Marra, S. Eddy, L. Hillier, et al. 1997. Washington University Zebrafish EST Project. GenBank ${ }^{\circledR}$ accession no. AA542678.

7.Fahrenkrug, S.C., M.O. Dahlquist, K. Clark, and P.B. Hackett. 1999. Dynamic and tissuespecific expression of eIF4E during zebrafish embryogenesis. Differentiation 65:191-201.

8.Fahrenkrug, S.C., B. Joshi, P.B. Hackett, and R. Jagus. 2000. Alternative transcriptional efficiencies and splicing define the translational efficiencies of zebrafish eIF4E mRNAs. Differentiation 66:15-22.

9.Gietz, D., A. St. Jean, R.A. Woods, and R.H. Schiestl. 1992. Improved method for high efficiency transformation of intact yeast cells. Nucleic Acids Res. 20:1425.

10.Gingras, A.-C., B. Raught, and N. Sonenberg. 1999. eIF4 initiation factors: effectors of mRNA recruitment to ribosomes and regulators of translation. Ann. Rev. Biochem. 8:913-963.

11.Jankowska-Anyszka, M., B.J. Lamphear, E.J. Aamodt, T. Harrington, Z.E. Darzynkiewic, R. Stolarski, and R.E. Rhoads. 1998. Multiple isoforms of eukaryotic protein synthesis initiation factor $4 \mathrm{E}$ in Caenorhabditis elegans can distinguish between mono- and trimethylated mRNA cap structures. J. Biol. Chem. 273:10538-10542.

12.Keiper, B.D., B.J. Lamphear, A. Deshpande, M. Jankowska-Anyszka, E. Aamodt, T. Blumenthal, and R. Rhoads. 2000. Functional characterization of five eIF4E isoforms in Caenorhabditis elegans. J. Biol. Chem. 275:10590-10596.

13.Lasko, P. 2000. The Drosophila melanogaster genome: translation factors and RNA binding proteins. J. Cell Biol. 150:F51-F56.

14.McKendrick, L., S.J. Morley, V.M. Pain, R. Jagus, and B. Joshi. 2001. Phosphorylation of eukaryotic initiation factor 4E (eIF4E) at Ser209 is not required for protein synthesis in vitro and in vivo. Eur. J. Biochem. 268:53755385.

15.Mumberg, D., R. Muller, and M. Funk. 1995. Yeast vectors for the controlled expression of heterologous proteins in different genetic backgrounds. Gene 156:119-122.

16.Ptushkina, M., K. Berthelot, T. von der Haar, L. Geffers, J. Warwicker, and J.E. McCarthy. 2001. A second eIF4E protein in Schizosaccharomyces pombe has distinct
eIF4G-binding properties. Nucleic Acids Res. 29:4561-4569.

17.Raught, B., A.-C. Gingras, and N. Sonenberg. 2000. Regulation of ribosomal recruitment, pp. 245-294. In N. Sonenberg, J.W.B. Hershey and M.B. Mathews (Eds.), Translational Control of Gene Expression. CSH Laboratory Press, Cold Spring Harbor, NY.

18.Robalino, J., B. Joshi, S.C. Fahrenkrug, and R. Jagus. Two zebrafish eIF4E family mem bers are functionally divergent and are differentially expressed. J. Biol. Chem. (Submitted.)

19. Robalino, J., B. Joshi, and R. Jagus. 2000. Danio rerio eukaryotic translation initiation factor eIF4E-1 mRNA, complete cds. GenBank accession no. AF257519.

20.Rodriguez, C.M., M.A. Freire, C. Camilleri, and C. Robaglia. 1998. The Arabidopsis thaliana cDNAs coding for eIF4E and eIF(iso)4E are not functionally equivalent for yeast complementation and are differentially expressed during plant development. Plant J. 13:465-473.

21.Rom, E., H.C. Kim, A.C. Gingras, J. Marcotrigiano, D. Favre, H. Olsen, S.K. Burley, and N. Sonenberg. 1998. Cloning and characterization of 4EHP, a novel mammalian eIF4Erelated cap-binding protein. J. Biol. Chem. 273:13104-13109.

22. Ruud, K.A., C. Kuhlow, D.J. Goss, and K.S. Browning. 1998. Identification and characterization of a novel cap-binding protein from Arabidopsis thaliana. J. Biol. Chem. 24:1032510330.

23.Sikorski, R.S. and J.D. Boeke. 1991. In vitro mutagenesis and plasmid shuffling: from cloned gene to mutant yeast. Methods Enzymol. 194:302-318.

24.Stern, B.D., M.A. Wilson, and R. Jagus. 1993. Use of non-reducing SDS-PAGE for monitoring renaturation of recombinant eIF4E. Protein Expr. Purif. 4:303-311.

25. Vasilescu, S., M. Ptushkina, B. Linz, P.P. Muller, and J.E. McCarthy. 1996. Mutants of eukaryotic initiation factor eIF-4E with altered mRNA cap binding specificity reprogram mRNA selection by ribosomes in Saccharomyces cerevisiae. J. Biol. Chem. 271:70307037.

26. Wach, A., A. Brachat, R. Pohlmann, and P. Philippsen. 1994. New heterologous modules for classical or PCR-based gene disruptions in Saccharomyces cerevisiae. Yeast 10:17931808.

27.Zhang, Y., H.L. Klein, and R.J. Schneider. 1995. Role of Ser-53 phosphorylation in the activity of human translation initiation factor eIF$4 \mathrm{E}$ in mammalian and yeast cells. Gene 163:283-288.

Received 27 August 2001; accepted 19 February 2002.

Address correspondence to:

Dr. Bhavesh Joshi

Center of Marine Biotechnology

University of Maryland Biotechnology Institute

Suite 236 Columbus Center

701 East Pratt Street

Baltimore, MD 21202, USA

e-mail: joshi@umbi.umd.edu 